\title{
RELACIONES A TRAVÉS DEL LENGUAJE: GERTRUDE STEIN Y ROBERT DUNCAN
}

\author{
Manuel Brito \\ Universidad de La Laguna
}

El acercamiento de Robert Duncan a la obra de Gertrude Stein supone para él el desarrollo de una poesía donde tengan cabida todos los elementos que le sean pertinentes en el momento del acto creativo. Y ello, principalmente, en lo que respecta a su deseo de no estar sujeto a reglas formales prefijadas, que puedan derivar de sus intensas lecturas literarias. Supone también liberar su imaginación y observar que el lenguaje posee suficiente capacidad como para provocar múltiples asociaciones.

Los primeros contactos que mantuvo Robert Duncan con la obra de la novelista y poetisa norteamericana datan de su época de estudiante en la universidad de Berkeley. Es aquí donde empezó a interesarse por los escritos steinianos debido, fundamentalmente, a la influencia de Robert Bartlett Haas y Louise Antoinette Krause. En especial el primero de ellos, quien se encontraba por esta época, finales de los años treinta, preparando una tesis doctoral sobre la obra de Stein y quien, además, posteriormente se convertiría en editor de su obra con libros como A Primer for the Gradual Understanding of Gertrude Stein (1971), Reflection on the Atomic Bomb (1973) y How Writing is Written (1974). El mismo Duncan declara años más tarde la importancia de ese encuentro con Haas quien llegó a posibilitar la identificación instantánea de Duncan con las formas literarias que propugnaba Stein, "So one afternoon he played me the records or Gertrude Stein reading, and loaded me with Stein to read, and my immediate response was to start writing in a steinian manner"'. Junto a este hecho, también se produce la coincidencia de que en 1937 Robert Duncan publica en la revista literaria

' Robert Duncan en la entrevista que sostuvo con Michael André Bernstein y Burton Hatlen, "Interview with Robert Duncan", Sagetrieb, vol. 4, n. 2/3, pág. 101. 
de la universidad de Berkeley, Occident, un poema titulado "People" junto a un pequeño relato de Stein que Robert B. Haas logró editar por primera vez, "Is Dead", el cual sería posteriormente reeditado por él mismo en How Writing Is Written. Pero, en cualquier caso, fue en la revista Epitaph donde aparecen los primeros textos duncanianos con claras influencias que se derivan de la técnica de Stein. Aquí podemos apreciar la utilización de un lenguaje que nos lleva a reconsiderarlo en sí mismo y al que nos vemos obligados a volver para aclarar y precisar mucho más el juego que crean los retruécanos, las repeticiones y las asociaciones que se van estableciendo. Para ejemplificarlo, podemos extraer un fragmento de un pequeño texto publicado en Epitaph y que lleva por título "Relativity, a love letter and relative to what; a love letter":

Suddenly one comes to be one living and one ceases to be one studying to be living. Suddenly one comes to be one living another one outside. Always one is living another one inside entirely, and sincerely another one being outside. Another is one inside who is outside.

This one is entirely this one. There are many ones and this one is entirely and sincerely this one ${ }^{2}$.

Aquí podemos encontrar las caracteristicas típicas que adornan el estilo de Stein como, por ejemplo, la utilización del pronombre «one» que viene a permitir una falta de concreción en cuanto a la persona que se refiere y que Duncan aprovechaba en esta carta de amor para dirigirse a su amante masculino, ya que él veía en su alrededor reacciones negativas ante sus sentimientos homosexuales, tal como confiesa en la entrevista mantenida con Michael A. Bernstein y Burton Hatlen ya citada. Por otra parte, también encontramos en este pequeño fragmento otras características steinianas, como la repetición que vemos aquí en la forma de participio presente de living y studying, o formas adverbiales como entirely y suddenly e incluso reincidencia de las mismas estruturas sintácticas donde el verbo to be juega un papel preponderante. Hechos éstos que podemos apreciar claramente en algunos de los retratos que Stein llevaba a cabo, como en este de Picasso:

This one was not completely working. This one was one not ever completely working. This one was not one working to have anything come out of him.

2 Robert Duncan, «Relativity a love letter and relative to what; a love letter», Epitaph, vol. I, n. ${ }^{\circ}$ I, pág. 21 . 
This one did have something having meaning that did come out of him. He always did something come out of him. He was working, he was not ever completely working. He did have some following him. He was one who was working. He was one having something coming out of him something having meaning. He was not ever completly working ${ }^{3}$.

Estos son, en consecuencia, los primeros indicios que podemos constatar del interés que Duncan por la técnica de Stein. Él se sentía atraído, en un principio, por lo extraño que resultaba el efecto que contenía ese juego con el lenguaje que podía dar lugar a hechos o conocimientos no esperados y que él, posteriormente, completaría y perfeccionaría cuando se relaciona con las teorías de Charles Olson, las cuales le sirven de estímulo para convertirse en un receptor inmediato de todo aquello que llegue en el momento del acto creativo, casi convirtiendo el poema en una aventura de reconocimientos. No obstante, durante la década de los años cuarenta no encontramos textos que confirmen la plasmación de este interés por la autora norteamericana autoexiliada en París, como lo demuestra el volumen de poemas The First Decade (1968) dedicado a la recopilación de poemas de estos años. Sin embargo, Jayne L. Walker afirma en su artículo, "Exercises in Disorder: Duncan's Imitations of Gertrude Stein", que el propio Duncan le confesó que en esa época seguía teniendo inclinaciones por la obra de Stein y, de hecho, adquirió y leyó numerosos textos de ella ${ }^{4}$.

Realmente fue durante los años cincuenta cuando de verdad se cimenta la relación entre Robert Duncan y Gertrude Stein. En las notas biográficas que escribió para la antología The New American Poetry editada por Donald Allen, él mismo nos recuerda esa proclividad hacia la obra de Stein ya que se estaba cuestionando en lo literario "the whole basis of an unbroken continuum in poetic language and tried to force a new sense of interrupted movement ${ }^{5 "}$. Duncan viene a reconocer que desde esta época ya tendía a la fragmentación, al detenimiento en una parte del discurso que llega a subvertir la lógica esperada que podemos hallar en los textos convencionales.

Las imitaciones que Robert Duncan lleva a cabo del estilo de Gertrude Stein están localizadas preferentemente en los años que van desde 1951 a 1955 , aunque no aparecen publicadas hasta la década de los años sesenta.

${ }^{3}$ Gertrude Stein, Look At Me Now and Here I Am, Penguin, Hammondsworth, 1971, pág. 215. A partir de este momento utilizaremos la nomenclatura $(L M N)$ para indicar este libro y hacer más fácil su referencia.

4 Jayne L. Walker, «Exercises in Disorder: Duncan's Imitations of Gertrude Stein», Robert J. Bertholf e Ian W. Reid eds., Robert Duncan. Scales of the Marvelous, New Directions, New York, 1979, pág. 24.

s Robert Duncan, «Biographical Notes», Donald M. Allen ed., The New American Poetry, Grove Press, New York, 1960, pág. 434. 
Las primeras en aparecer son aquellas concebidas originariamente como un libro independiente bajo el título de Writing Writing (1964). Escritas en el año 1953 y con la intención, según confiesa a Walker, de ser una respuesta al libro de Stein How to Writing. Son éstas unas imitaciones donde encontramos no sólo poemas sino también problemas afines a la poética que intentaba desarrollar. En 1967, dentro de la revista Audit, que le dedica un número especial, llega a publicar dos poemas del mismo estilo steiniano, como son "The King: A Regret y Cloudy", bajo la denominación general de "Uncollected Stein Imitations from the period of Writing' Writing" (1953). Ya posteriormente aparece una recopilación más amplia en una edición que llevó a cabo la editorial londinense Fulcrum Press en un volumen cuyo título Derivations (1968) también acoge a Letters, un libro de poemas que se había publicado previamente en 1958. En Derivations nos encontramos con aquellas Imitations of Gertrude Stein redactadas entre 1951 y 1952, algunas partes de Writing Writing y con la segunda parte de Imitations of Gertrude Stein pertenecientes a los años que van desde 1953 a 1955. En 1969 también aparece Play Time Pseudo Stein donde se encuentran algunos poemas imitando a Stein, especialmente en cuanto a la repetición de palabras y utilización del retruécano e incluyendo, asimismo, una pequeña comedia titulada $A$ Fairy Play, todos estos textos pertenecientes al año 1953.

La identificación durante estos años cincuenta con la técnica steiniana sirvió a Duncan fundamentalmente para explorar las posibilidades que otorga el lenguaje como medio adecuado para el conocimiento de sí mismo y también como asentamiento definitivo en la idea de que el acto creativo está siempre en proceso de descubrimiento. Son ideas que conectarían más tarde con aquellas propiciadas por Olson y las encontradas en el filósofo inglés Alfred North Whitehead. Jayne L. Walker resume estos años de identificación entre Duncan y Stein de manera precisa, definiéndonos concisamente estos años de acercamiento entre los dos:

His apprenticeship to Stein taught him a new attention to the commitment to writing as a temporal, sequential process. He continued to use verbal patterning, as she did, to "represent" directly the movements of consciousness in language ${ }^{6}$.

El acercamiento de Robert Duncan a las técnicas propiciadas por Gertrude Stein, y que él desarrolla principalmente en la primera mitad de los años cincuenta, se basa fundamentalmente en la consideración de que el

- Jayne L. Walker, op. cit., pág. 34. 
lenguaje tiene suficiente entidad por sí mismo. Es decir, tratarlo como una realidad que refleje la imaginación del poeta. Las palabras que aparecen en el poema no necesitan de la reflexión o interpretación racional-discursiva sino que requieren la identificación o comunión con ellas. En teoría, las palabras no se remiten a lo que significan sino a sí mismas para que entremos en contacto con ellas y detengamos nuestra mirada en ellas. Para Duncan, la poesía de Stein, que es donde ella únicamente permite la utilización de sustantivos y que se caracteriza por su intensidad y detenimiento en cada uno de los elementos del verso, es una poesía que contiene todo el sufrimiento y el placer de la lectura, 'pero en la que no existe el hábito normal de la conclusión. Los significados aparecen y desaparecen, es una forma abstracta que se va moviendo y creando a sí misma. Las palabras se transforman y existen con diferentes significados para cada lector, ya que Stein tiene en cuenta las vastas posibilidades de la percepción en cada individuo. En las «Imitations of Gertrude Stein» de Robert Duncan tenemos suficientes poemas donde la palabra toma su papel preponderante y, para ello, llega incluso a dejarlas en solitario con el fin de que las consideremos y cuestionemos por sí mismas. Así, por ejemplo, lo lleva a cabo en su poema "Poetry permit for volley". Este poema se caracteriza principalmente por la utilización de frases muy cortas que reverberan unas sobre otras por mor del retruécano, para terminar fijándonos en cada una de las palabras finales de manera obligada al formar cada una de ellas una realidad independiente:

\author{
Palaces catches. Palaces started. Catches \\ places to place. \\ Fallacies to face. Coaches to rent. \\ Places to go to. I cannot. \\ You go to. I do not want. \\ Thay will want to hear the song to sing. \\ All. The. Way. Thru?.
}

También en otro poema de esta serie como es "Dance early spring weather magic", la palabra asume el rol principal. El tema gira en torno al aliento que da el poeta a unos jóvenes para que bailen y disfruten del contacto físico y, al mismo tiempo, se muestren un amor gozoso. La repetición fre-

Robert Duncan, Derivations. Selected Poems 1950-1956, Fulcrum Press, London, 1968, pág. 29. A partir de ahora vamos a utilizar la abreviatura $D$ para este libro y $W W$ para $W$ riting Writing. A Composition Book. Stein Imitations, Sumbooks, Alburquerque, 1964. En estos dos libros podemos encontrar recopilada la mayoria de las imitaciones de Gertrude Stein. 
cuente y continua de palabras como hop y hug nos introducen en el ambiente de rapidez e inmediatez de la acción, pero también nos obliga a volver una y otra vez sobre las palabras, haciendo que nos detengamos en ellas, junto a esto nos vemos inmersos en el torbellino propio del baile al utilizar repetidamente una palabra como round:

Hug the girls, boys. Hug the boys, boys.

Hug the girls, girls. Hug the boys, girls.

Round, and around and round.

Remind me. Remind me.

Hop to a hug, hop. Hug. Kiss

the girls, boys. Kiss the boys.

$O$ boy, boys. Kiss the boys, girls.

Round, around and round.

Hop to the boys, boys. Hear the tune

boys. Hug the girls, girls.

Hop with the girls as the tune twirls

girls hop with the boys now.

How. Hop. And now. Hop. (D, 35)

Este ritmo steiniano caracterizado por la utilización de un lenguaje sencillo va en consonancia con una acción común como es la del baile y donde se combina, a causa de la fragmentación, la simplicidad y abstracción de éste. W. H. Gass, refiriéndose a Gertrude Stein relaciona esta técnica con la pintura moderna, especialmente el cubismo y el expresionismo, al sugerir realidades eufemísticas como es la disociación, la confusión o la sorpresa, lo que nos lleva a considerar a la palabra por su propia realidad como ocurre también en el caso de Duncan:

...but she was often able to another step, the last available to protective speech: that of giving to her words the feelings that arise from things; that of creating from her words real objects, valuable for themselves, capable of an independent existence, as physical as statuary ${ }^{\mathrm{B}}$.

${ }^{\circledR}$ W. H. Gass, Gertrude Stein: «Her Escape from Protective Language», Accent, n. ${ }^{\circ}$ 18, pág. 240. 
Duncan no se detiene exclusivamente en este punto sino que también experimenta con el juego que pueden proporcionar las palabras. Como realidades que son en sí mismas también pueden generar sus propias derivaciones. Concretamente, en su poema "Spanish Lessons", curiosamente el último de las Imitations of Gertrude Stein, nos hace ver cómo la palabra se transforma tanto lingüísticamente como en el simple nivel fónico:

A bocadillo is a little bite. Mouths are bocas.

Moths are polillas.

Everywhere mosquitos are there.

In verano. Es en verano.

Heat herd calor for color.

Hace mucho calor. De rojo?

Tostado?

Interest. Ing. An interest. Ing.

Ing. Ing. $(D, 86)$

El juego con el sonido proviene claramente de la introducción de palabras españolas que se llegan a fundir con las inglesas, como ocurre en el caso de calor y color, que permiten el equívoco y el libre juego asociativo de sus significados respectivos. Ya Cary Nelson nos avisa de que este juego puede resultar frívolo pero que la intención de Duncan es clara al hacer que nos dejemos guiar en parte por la irracionalidad, para mantener "our faith in unconscious decisions" a la vez que nos sintamos perdidos por este "flowering sound" ". Duncan también juega con la lingüistica cuando hace que nos fijemos en la parte de una palabra, como ha ocurrido con la partición que se ha hecho de "interesting". La fragmentación o incoherencia parece evidente, lo cual es más relevante si un lector inglés o español no conoce el otro idioma siendo, entonces, el sonido o palabra que lee los que generan claramente sus propias emociones y no la realidad a la que hace referencia. Hasta cierto punto, el lenguaje se ha liberado de su control racional y tenemos que es la escritura la que ha hallado su propia expresión.

En consecuencia, tenemos un logocentrismo que puede proporcionar una pluridimensionalidad en este tipo de literatura fragmentaria y experimental. El mismo Duncan desarrolla este aspecto poético, en cuanto a la importancia de la escritura como tal, principalmente en Writing Writing donde hallamos pequeños ensayos como «The Code of Justinian. A Dis125.

${ }^{9}$ Cfr. Cary Nelson, Our Last First Poets, University of Illinois Press, Urbana, 1981, pág. 
course on Justice», "The Feeling of Language in Poetry», "Writing As Writing» y el titulado «6/16/53». Creemos que existen dos aspectos que unen a estos cuatro textos. El primero de ellos es la importancia de los sentidos en la percepción del poeta, que se convierten en un medio directo e inmediato de aquellos elementos que recibe la imaginación. Y, en segundo lugar, una cierta atracción por dejar que surja lo impredecible y, lógicamente, potenciando la parte inconsciente. Así, por ejemplo, tenemos que en «The Code of Justinian. A Discourse on Justice», se deja obedecer por una escritura que se regula a sí misma, que se ordena y progresa de acuerdo con su propio diseño. El escritor pertenece a esa escritura y, al no atenerse a ninguna regla que lleve a una conclusión determinada, puede dar lugar a un cierto sentido irracional. Cuando él mismo afirma "writing is like writing" ( $W W$, sin paginar) confirma la supremacía del acto de escribir, donde el escritor se debería limitar a oír la melodía poética, una idea poética ésta que desarrollaría posteriormente cuando conecta con las ideas de Olson y las ejemplifica en la serie Passages, dejándose guiar por las voces de la "muse", o la del "Master of Rime" en su serie The Structure of Rime.

Pero es en Writing As Writing donde llega a unificar los sentidos con la ambigüedad que posee la palabra en sí misma, si se expone de manera fragmentada. Aquí la presentación de los sentidos se lleva a cabo mediante la paradoja de asignarle los órganos que no le corresponden, "The word in the hand is the sound in the eye is the sight in the listening mind". (WW, sin paginar), lo cual parece tender a poner de relieve una mayor interrelación entre todos ellos. Al lado de esto, tenemos a la palabra que se incluye dentro del flujo del tiempo y que se caracteriza por la posibilidad del equívoco, la fragmentación y una visión nueva que nos lleva a la originalidad:

The word at rest rests in the mind in the restless continuation. The breaking down of all internal continual. The interruption of persistent locomotion. The persistent irruption of volcanic inconsequence. The landscape revised to portray a reality. ( $W W$ sin paginar.)

Esta es una idea que conecta con Stein en el sentido de que no tenemos por qué seguir una línea marcada, ni buscar la causalidad, sino introducirnos en la esencia del lenguaje; un hecho al que William Carlos Williams hace referencia cuando habla de Gertrude Stein. El poeta incide en ese aspecto de la autora norteamericana donde las palabras parecen transcender todo:

What are philosophers, scientists, religionists, they that have filled up lite- 
rature with their pap? Writers, of a kind. Stein simply erases their stories, turns them off and does without them, their logic (founded merely on the limits of the perceptions) which is supposed to transcend the words, along with them. Stein denies it. The words, in writing, she discloses, transcend everything ${ }^{10}$.

Por último, en "The Feeling of Language in Poetry" y "6/16/53", Duncan sigue reafirmando la intervención de los sentidos en el proceso creativo que llega a ilustrar nuestros mecanismos mentales, nuestra manera de percibir y la manera en que vivimos. La escritura, en definitiva, refleja nuestras metas y nuestra multiplicidad interior. Al mismo tiempo y finalmente, el lenguaje posibilita una reordenación, ya que no es "but a hut of words primitive to our nature" y al que se llega sabiendo escuchar la melodía que proyecta y las interconexiones que proyecta, "his poetry pictures his listening" ( $W W$, sin paginar). Este deseo de que sea el propio lenguaje el que se manifieste de forma significativa es análogo al mismo que expresa Stein en How To Write cuando se refiere a la frase. Para ella, los elementos de la frase están siempre con el escritor, sin necesidad de que se remitan a un desarrollo lógico y fijándose en la frase en sí:

A sentence makes it be just as well that they will be theirs as a pleasure. A sentence makes a very little impression particularly if it has been doubly prepared. In this way they will arrange that they move around. A pleasure is their pleasure. This is a sentence in this respect. At once. A sentence is not caught they will be as well as directed with their outline".

Esta idea steiniana de someterse al propio curso que lleva el lenguaje, y a la que se apega Robert Duncan, podríamos considerarla relacionada con el concepto de "process" en Whitehead que estudiaremos en un capítulo posterior. Duncan desarrollará el sometimiento del escritor al tiempo presente de un modo diferente a Stein. Él va a seguir más bien a Whitehead $\mathrm{y}$, a grandes rasgos, ese momento inmediato y presente será sólo un instante que es resultado de un pasado e incidirá en el futuro. Stein se inclina más por el estaticismo de ese momento, profundizando y volviendo sobre él, estableciendo un proceso lógico a través del cual se puede conocer mejor la conexión entre perceptor y objeto percibido. Duncan, incluso, va a permitir

10 William Carlos Williams, «The Work of Gertrude Stein», en Donald M. Allen y Warren Tallman eds., The Poetics of the New American Poetry, Grove Press, New York, 1973, pág. 134.

"Gertrude Stein, How To Write, Something Else Press, Barton, 1973, pág. 134. 
que la palabra, una vez elaborado el poema, pueda ser conectada y contrastada con las significaciones que ha tenido a lo largo de la historia.

Pero, en esta época de principios de los años cincuenta, Duncan sí estaba interesado en presentarnos el funcionamiento de la mente y su relación con el lenguaje. Hasta tal punto esto es así, que nos encontramos con poemas como "Several Poems. In prose", donde cada verso se puede ver como perteneciente a un poema global o puede considerarse de manera autónoma. La mayoría de los versos consta de una sola frase y se encuentran aislados del resto por una separación de cuatro versos en blanco y un punto en medio de ellos. No existe una idea progresiva o un punto referencial en el que apoyarnos, simplemente se nos presentan pensamientos inmediatos que se caracterizan por la incoherencia entre ellos. Ya el primer verso nos formula una pregunta directa que no contestaremos al final, "Does this mean a meaning?" $(D, 82)$. Las mismas interrogaciones que se hacen hacia la mitad del poema se deberían sostener por sí mismas, "Can you derive pleasure?, What counts as counting?, If you cannot see through it, can you see through it?" $(D, 82)$, son frases instantáneas cuyo poder asociativo se multiplica de acuerdo con la experiencia de cada uno. Para completar lo anterior, el segundo verso menciona a Shakespeare, "After Shakespeare there was pleasure in prose" que le va a permitir un juego de palabras inmediato utilizando, para ello, el francés junto al inglés, "Shake: spear, spare or peer. Il $n$ y a pas de pére/ who is his peer. This pair". $(D, 82)$ Este poema viene a reflejar la instantaneidad con que el autor se acerca al acto creativo; se imbuye en un proceso donde no hay prefijaciones y nos presenta simplemente frases o fragmentos que aparecen como ráfagas de la mente. Duncan se acerca, de este modo, al mismo proceso que seguía Stein y que Neil Schmitz ha definido certeramente:

Everything is contingent, changing as it moves and the mind moves. To write in this mode of perception, Gertrude Stein constantly presses against the order of language those elements of syntax and signification that provide philosophical and scientific discourse with its stability. Judged by its criteria, she is indeed senseless ${ }^{\prime 2}$.

Duncan es consciente durante esta época del cambio constante a que se ve sometido dentro del tiempo y de la incoherencia que puede derivarse de ello si se fija aisladamente en los diversos elementos que componen la experiencia. Pero este mismo hecho le sirve para descubrir nuevos aspectos y

12 Neil Schmitz, "Gertrude Stein As Post-Modernist: The Rhetoric of Tender Buttons», Journal of Modern Literature, vol. 3, n. ${ }^{\circ}$, pág. 1.206. 
situaciones no esperadas que también sirven como elemento renovador de su poesía. Desea que lo directo y lo inmediato se conviertan en un hábito, tal como afirma en otro poema de estas "Imitations of Gertrude Stein":

Follow the line and profit by the width of this course.

This the winning thru a discourse.

$A$ continual habit. $(D, 76)$

Otra de las técnicas steinianas utilizadas por Robert Duncan como artificio rítmico es la repetición. A Stein no le gustaba hablar de repetición, tal como afirma en Portraits and Repetitions, sino más bien de insistencia. Es decir, poner énfasis, utilizando para ello el "beginning again and again", para poder describir denotativamente toda la realidad de la "thing seen". En realidad, no puede haber repetición literal porque existe el movimiento dentro del tiempo y no se puede volver a producir la misma realidad de igual forma a como sucedió por haber cambiado los condicionamientos. Robert Duncan roza este punto de vista que preconizaba Stein pero también utiliza la repetición como un artificio rítmico formal que coadyuve a nuestra fijación en el lenguaje:

Beginning tyo write. Continuing finally to write. Writing finally to continue beginning.

To overcome the beginning. To overcome the urgency. To overcome writing in writing.

Not ever to overcome the beginning. Now to write writing. Not to overcome in beginning. ( $W W$, sin paginar.)

En otros poemas también utiliza la sutileza de un tempo rítmico de manera más evidente, donde la repetición constante de una forma verbal va creando diversos efectos. Así, por ejemplo, en los poemas "Turning Into, Coming Out Of y Making Up" que encontramos en Derivations o el que se titula "I'm not Afraid" de Writing Writing, las tres formas de gerundio y la de presente, I'm Not Afraid, se repiten alineadamente en todos los versos, implicando con ello la insistencia de las cuatro acciones que indican estos cuatro verbos. Lo importante de repetir mecánicamente estas formas ver- 
bales es que nos lleva a conocer los deseos o anhelos particulares suyos de un modo ritual $\mathrm{y}$, al mismo tiempo, va definiendo con más precisión los límites de su personalidad y psicología. Al lado de esta repetición de palabras que se produce automáticamente al comienzo de cada verso (también se puede producir al final del verso como ocurre en el poema "Division" de Writing Writing con la palabra "killing'), tenemos la repetición de una palabra y sus correspondientes variaciones que proporcionan efectos sonoros similares, como hemos apreciado en algunos de los retruécanos ya estudiados. Éstos son, por ejemplo, los que llevan a Warren Tallman a definir la primera etapa de Duncan, antes de su adentramiento en el concepto de "open field", en estos términos, "All is precise, yet almost dreamy, accurate yet enchanted"13. En estos últimos poemas es donde se manifiesta la mayor inventiva de Duncan, demostrándonos que no sólo la palabra tiene su realidad sino que el poeta se siente unido y devoto de ella, ofreciéndonos, mediante la concentración del énfasis en ella, todo lo que denota y connota como sucede en su poema Cloudy:

Passages in time or clouds as in passing.

Clouds as in a Tiepolo scene. Clouds

in tableau, a table-d'hote pass ring.

A Skirt. A scattered. Magic. In scattering.

Scatter, a ring of clouds about a scant, cloud

as scatter.

Pose a soft center solid in visibility. Dense it delivers itself out of its pose. A position in drifting.

Swift cloud sails westward solid and soild. Swift westward silently sails. This is running across the sky without arranging itself. As a tree blows in cloudy skirts and rustles. Passion, a time of swift indecisions, a milk lets us see the containing glass, drops on the table show soils of the time. Passing the time its indecisions. ${ }^{14}$

Duncan se interesa por alejarnos de una repetición vacía donde los pen-

${ }^{13}$ Warren Tallman, The Eternal Mood: Robert Duncan's Devotion To Language", Open Letter, n. ${ }^{\circ} 6$, pág. 71 .

${ }^{14}$ Robert Duncan, Cloudy, Audit, n. ${ }^{\circ} 4$, pág. 34. 
samientos no se vayan a relacionar con el principal concepto que intenta desarrollar, como es el del tiempo. Nos viene a ofrecer la importancia de la experiencia que se ha vivido con todos los movimientos perceptuales que se han adquirido a través de ella. Para esto, se apoya en la referencia a Tiépolo o en las características propias de las nubes como puede ser el rumbo que toman, su carácter disperso o su contacto con el cielo y la tierra, estableciendo, definitivamente, la transitoriedad del tiempo al relacionarlo con ellas.

La repetición también queda manifiesta en otro poema de esta serie, $A n$ Arrangement, donde la palabra locomotive se va transformando atendiendo aparentemente a los sonidos de un tren en marcha, sin una sintaxis que nos remita a un plan en progreso que deba llegar a una conclusión definitiva:

\author{
loco-co co moto mo mo \\ locomomo cotivecomo \\ mocolotive \\ motocolive \\ moco lomo motive lomo \\ co co co co co como co momo \\ lo mo como lo mo tomo \\ At Lake Como we saw mountains. (D, 33).
}

En todo caso, podríamos llegar a una conclusión denotativa que es la que nos ofrece sugerentemente Hugh Kenner, que vuelve a incidir en la tarea imaginativa que ha llevado a cabo Duncan y de la que se nutre claramente durante el proceso perceptual:

The way he took the word alocomotive» apart: that's the way he's treating three or four scenes we all have in our minds: herdsmen and animals; the melodramatist's parlor set; the Cro-magnon artist. His materials have the status of found objects, easily retrieved from imagination's bric-á-brac. ${ }^{15}$

En lo que respecta al nombre o sustantivo, Robert Duncan coincide con 25.

is Hugh Kenner, "1680 Words on Robert Duncan's Words", Ironwood 22, vol. 11, 2, pág. 
Gertrude Stein. Ella rechazaba los nombres en la prosa pero consideraba que la poesía debería incluir todo y creía natural que, al incluir todo lo que sucedía, ello podía hacerse real a todos los lectores especificando por su nombre literal lo que sucedía. Los ejemplos que nos ofrece Stein en "Poetry and Grammar" como Homero, Chaucer o la misma Biblia, sirven para demostrar que los diversos autores usaban y sentían el nombre porque, para ella, la poesía es esencialmente el amor y la pasión por un nombre en concreto. Es decir, establece una relación estrecha con el modo natural en que uno se expresa cuando amamos una cosa y como, para ella, manifestamos nuestros sentimientos o emociones de manera intensa y densa, entonces se produce una menor longitud de la frase dando lugar al verso:

Think of all that early poetry, think of Homer, think of Chaucer, think of the Bible and you will see what I mean you will realize that they were drunk with nouns, to name to know how to name earth sea and sky and all that was in them was enough to make them live and love in names, and that is what poetry is it is a state of knowing and feeling a name... Think what you do when you do that when you love the name of anything really love its name. Inevitably you express yourself in that way, in the way poetry expresses itself that is in short lines in repeating what you began in order to do it again. ( $L M N$, 139-140).

De igual modo, Duncan emplea con profusión el sustantivo en los textos poéticos de esta época y su concentración está dirigida, normalmente mediante recursos formales (como el dejarlo aislado para que nos fijemos en él), a su propia existencia y al proceso que sigue el nombre en reciprocidad con su propia consciousness. El nombre en la poesia de Robert Duncan adquiere toda su hegemonía y no sólo proporciona el sentido tangible que conlleva sino que nos remite, mediante su aislamiento e insistencia, a impresiones posteriores que podamos suponer o saber con certeza, dependiendo de las referencias disponibles. El nombre en su poesía sí que adquiere toda su etimología e historia. Quizás donde quede más aclarado este concepto sea cuando llega a la utilización de nombres propios; tanto en el caso de Gertrude Stein como en Duncan la cuestión adquiere claras semejanzas. En una de las partes de The Autobiography of Rose de la autora norteamericana, los versos que encontramos se van a detener especialmente en dos nombres propios, Allan y Rose, principalmente en esta última. La escritura experimental de Stein parece llevarnos a lo ininteligible o incoherente incluso dentro de su poesía donde, a pesar de utilizar nombres propios o sustantivos, cada uno de ellos se puede multiplicar y fragmentar: 
Grass that is cut is hay.

There there is sunshine

Here there is snow

There there is a little boy

Here there is a little girl

There his name is Allan

Here her name is Rose

It is interesting.

Rose has an autobiography even if her name was not Rose ${ }^{16}$

¿A dónde quiere llegar Gertrude Stein? Quizás a transmitirnos el alejamiento de dos personas, que viene dado por las contraposiciones de "here" y "there" y las diferentes situaciones que se están produciendo en ambos sitios. En cualquier caso, nos remite una vez más, a pesar de la aparente concreción de los nombres (nunca lograda al no definirlos y desarrollarlos totalmente), a la independencia y relaciones simultáneas de experiencias que se dan en autor y lector. Stein intenta poner más de relieve la propia intensidad o psicologia de los personajes sin explicar para nada su comportamiento, dejando que trasluzca una cierta ambigüedad y misterio en esas relaciones para despertar connotaciones no esperadas. Es el mismo juego que sigue Robert Duncan cuando utiliza nombres propios como en el caso de su poema Names of People:

Names of people. Lili Fenichel.

Lili Fenichel. Names of people.

George Racket. Parker Parker.

Ambrose Bierce. Adrian Wilson.

Under the umbrella

Madeline, Madeline.

Into the sunshine

with Maripose Sampson.

The name of a person.

The name of a river.

Ham and Mary and Brenda Tyler

${ }^{16}$ Gertrude Stein, How Writing is Written, Robert B. Haas ed., Black Sparrow Press, Santa Barbara, 1977, pág. 39. 
came over for dinner.

Who will forget kindly Jack Spicer?

Who will forget Old Black Joe?

Who will forget the David Barys,

Who will forget Brock Brockway to show?

Under the umbrella

Mary Ellen Rae was lovely to name

Into the showtime, all time.

Know time

names of people. Lili Lili Fenichel. (D, 28)

Los nombres citados hablan por sí mismos y las sugerencias se multiplican en cada uno de ellos. Podemos conocer algunas referencias de ellos como, por ejemplo, aquellos relacionados con sus primeros años de formación poética como Mary, Ham y Brenda Tyler o Jack Spicer, también a Ambrose Bierce quien ejerció una indudable influencia en los ambientes literarios de San Francisco a finales del siglo XIX, pero Duncan los libera, como ocurría en el poema de Stein, de la anécdota y la descripción y cada uno de ellos demanda un detenimiento más preciso que nos llevará seguramente a nuevas asociaciones. No encontramos una conclusión definitiva que nos confirme una opinión acerca del ambiente o significación potencial de ellos y cuando se indica una acción nos la presenta de manera aséptica como ocurre en la cena con el matrimonio Tyler o la ubicación de Madeline bajo la sombrilla. A este hecho ayuda el nivel formal con que se nos ha presentado. En primer lugar, aislando algunos nombres y convirtiendo cada uno de ellos en una frase por sí mismos, como ocurre en el tercer y cuarto verso. En segundo término, también esto se ha llevado a cabo mediante la repetición de ellos; insistiendo en el nombre se ha logrado transmitir la importancia que tenían para él su personalidad (sería el caso de Lili Fenichel y Madeline). $Y$, en último lugar, nos presenta una misma interrogación repetitiva, "Who will forget", que la aplica a cuatro de ellos, como son Jack Spicer, Old Black Joe, David Barys y Brock Brockway.

La atracción de Robert Duncan por Gertrude Stein se basó en la búsqueda de una disciplina que le permitiera romper la sintaxis y la planificación lógica del texto y hallar una técnica que pudiera dar lugar a respuestas conscientes o inconscientes que se desarrollan en el tiempo. Pero creemos que hay una diferencia sustancial entre ambos y que se va a poner de manifiesto en la posterior trayectoria poética de Duncan. En Stein encontramos un intento de hacer más científica, si cabe, la literatura, con el fin de trasladar todos los elementos de la realidad, siguiendo patrones psicológicos o filosóficos ya apuntados por William James con el fin de conocer más profundamente la mente y realidad humanas. Stein, como apunta Allegra Stewart, no participaba de la transcendencia de la realidad, aunque creía 
en la armonia entre hombres y universo, bajo un punto de vista espiritual, sino que participaba completamente de la experiencia, "contemplating it, loving it, dreaming about it and expressing it in words" $" 17$. Sin embargo, Robert Duncan acepta la irracionalidad como medio de conocimiento, el que la imaginación se mueva por todos los sitios y traiga elementos que puedan provenir de fuerzas desconocidas, de símbolos o voces que le hablan durante el acto creativo, lo cual se verá especialmente reforzado después de su lectura del Process and Reality de Alfred N. Whitehead y sus ideas sobre la metafísica, que en Duncan se plasman en la yuxtaposición de numerosos elementos espirituales de los que se nutre, desde la Cábala a leyendas bíblicas pasando por el Zohar de Moisés de León.

En definitiva, la técnica adquirida de Stein le sirvió a Duncan para liberarse de tener que hacer una literatura coherente y abandonar las tensiones del discurso racional. Y lo que apreció fundamentalmente en Stein es esa "wondrous invention" que encontraba en esa "exuberant force enclosed inside the private parts of speech" como dice William Wasserstrom del estilo steiniano $^{18}$. Esto va a dar como resultado en la obra de Duncan el que se fije en la palabra, a la que otorga realidad autónoma, el dejarse guiar por los sonidos que ellas generen y aceptar definitivamente la ambivalencia que pueda prestar la fragmentación. Una postura eminentemente lúdica que él concibe en el seno de ese acto poético al que se entrega.

${ }^{17}$ Allegra Stewart, "The Quality of Gertrude Stein's Creativity», American Literature, vol. 28 March 1956, pág. 503.

18 William Wasserstrom, «The Sursymannercubealism of Gertrude Stein», Twentieth Century Literature, vol. $21, \mathrm{n} .^{\circ} 1$, pág. 106. 